\title{
Modelling and mathematical results arising from ferromagnetic problems
}

\author{
DESCLOUX Jean, FLUECK Michel* \& RAPPAZ Jacques \\ Department of Mathematics, Ecole Polytechnique Fédérale de Lausanne (EPFL), Lausanne 1015, Switzerland \\ Email: jean.descloux@epfl.ch, michel.flueck@epfl.ch, jacques.rappaz@epfl.ch \\ Received May 19, 2010; accepted June 23, 2011; published online October 18, 2011
}

\begin{abstract}
In this article, we investigate the equations of magnetostatics for a configuration where a ferromagnetic material occupies a bounded domain and is surrounded by vacuum. Furthermore, the ferromagnetic law takes the form

$$
\boldsymbol{B}=\mu_{0} \mu_{r}(|\boldsymbol{H}|) \boldsymbol{H},
$$

i.e., the magnetizing field $\boldsymbol{H}$ and the magnetic induction $\boldsymbol{B}$ are collinear, but the relative permeability $\mu_{r}$ is allowed to depend on the modulus of $\boldsymbol{H}$. We prove the well-posedness of the magnetostatic problem under suitable convexity assumptions, and the convergence of several iterative methods, both for the original problem set in the Beppo-Levi space $W^{1}\left(\mathbb{R}^{3}\right)$, and for a finite-dimensional approximation. The theoretical results are illustrated by numerical examples, which capture the known physical phenomena.
\end{abstract}

Keywords ferromagnetism, nonlinear elliptic problems, finite element methods, optimisation algorithms MSC(2010) 78A30, 35J65, 65M60, 74P99

Citation: Descloux J, Flueck M, Rappaz J. Modelling and mathematical results arising from ferromagnetic problems. Sci China Math, 2012, 55(5): 1053-1067, doi: 10.1007/s11425-011-4306-6

\section{Introduction}

We present in this article an application of stationary electromagnetics in the case of ferromagnetic materials related to magnetic shielding.

It is well known that, in a ferromagnetic material, the behaviour of the magnetic induction $\boldsymbol{B}$ in the function of the magnetic field $\boldsymbol{H}$ is nonlinear, and that $\boldsymbol{B}$ and $\boldsymbol{H}$ are not collinear in general, thus resulting in hysteresis phenomena. However, in most industrial applications, it is sufficient to consider $\boldsymbol{B}$ and $\boldsymbol{H}$ collinear, especially when the Euclidean norm of $\boldsymbol{H}$ (denoted by $|\boldsymbol{H}|$ ) is large. In this case, we have the relation

$$
\boldsymbol{B}=\mu_{0} \mu_{r}(|\boldsymbol{H}|) \boldsymbol{H},
$$

where $\mu_{r}$ is defined as the relative magnetic permeability, and is a positive, decreasing and convex function of the Euclidean norm of $\boldsymbol{H}$. The nonlinear mapping $\boldsymbol{B} \mapsto \boldsymbol{H}$ results in limiting the penetration of electromagnetic fields into a space. This effect is often referred to as electromagnetic shielding or screening of the ferromagnetic material.

A typical example takes place in the screening effect of the steel shell supporting a cell for aluminum production. Let us detail this particular case: in the aluminum industry, pure metal is produced in

*Corresponding author 
electrolytic cells by the electrolytic processing of alumina created by very strong stationary electric currents (several hundreds of thousands of Amperes). The liquid aluminum produced at the cell cathodes, and the electrolytic bath in which are placed the anodes, are submitted to intensive electromagnetic forces. The resulting effect is a motion of the liquids. In order to ensure the strength of the device, the lower and lateral cell faces are covered by steel plates called "the shell of the cell". The magnetic induction is shielded by these shells and, therefore, the Lorentz forces and minimized. Hence the liquid motion in the cells is smaller, which improves the stability of the process. In order to analyze the cell stability and compute the fluid flow, it is thus important to predict this screen effect.

Let us consider a bounded domain $\Omega$ in the three-dimensional space $\mathbb{R}^{3}$ occupied by a ferromagnetic material with relative magnetic permeability $\mu_{r}$ and let $\Omega^{\prime}:=\mathbb{R}^{3} \backslash \bar{\Omega}$ stand for the exterior domain. We assume that a stationary electric current with density $\boldsymbol{j}$ flows in a conductor surrounding $\bar{\Omega}$ and creates a magnetic field $\boldsymbol{H}$ and a magnetic induction $\boldsymbol{B}$. Following [3,7] we start with the derivation of the equation that governs the electromagnetic induction in terms of the magnetic field $\boldsymbol{H}$. An existence and uniqueness result for the obtained model is then given following the approach in [8-10]. The numerical solution by an iterative procedure is then addressed. We first derive a fixed point algorithm to solve the nonlinear problem by a sequence of linear problems to solve at each iteration. The analysis of the method is handled for the continuous problem and a domain decomposition technique is presented and analyzed. This technique allows to solve the problem only in a bounded region. Then, we present a finite element method for the approximation of the continuous problem, and we study the convergence of the iterative procedure for the discrete problem. Finally, numerical experiments performed in a realistic setup are presented.

A large number of publications are devoted to the mathematical modelling and the numerical solution to problems with absorbing ferromagnetic materials, with the Landau-Lifschitz law for propagation and scattering of electromagnetic waves. Let us mention $[13,14]$ and the references therein. Other papers address the magnetostatic problem on thin plates $[7,12]$ in which the thickness of the plate tends to zero when $\mu_{r}$ tends to the infinity.

\section{Modelling}

It is well known that if the domain $\Omega$ is a vacuum or is occupied by a non ferromagnetic material (i.e., without magnetic effects in $\Omega$ ), the field $\boldsymbol{H}$, denoted in this case by $\boldsymbol{H}_{0}$, can be described in function of $j$ by using the Biot and Savart law:

$$
\boldsymbol{H}_{0}(\boldsymbol{x})=\int_{\mathbb{R}^{3}} \boldsymbol{\nabla}_{\boldsymbol{x}} G(\boldsymbol{x}, \boldsymbol{y}) \times \boldsymbol{j}(\boldsymbol{y}) d y,
$$

where $G(\boldsymbol{x}, \boldsymbol{y})$ is the Green kernel given by

$$
G(\boldsymbol{x}, \boldsymbol{y})=\frac{1}{4 \pi|\boldsymbol{x}-\boldsymbol{y}|}, \quad \boldsymbol{x}, \boldsymbol{y} \in \mathbb{R}^{3} ;
$$

here $|\cdot|$ denotes the Euclidean norm. In this case, the induction magnetic field $\boldsymbol{B}_{0}$ corresponding to $\boldsymbol{H}_{0}$ is given by $\boldsymbol{B}_{0}=\mu_{0} \boldsymbol{H}_{0}$, where $\mu_{0}$ is the magnetic permeability of the vacuum, and the following relations hold in $\mathbb{R}^{3}$ :

$$
\operatorname{div}\left(\boldsymbol{B}_{0}\right)=0, \quad \operatorname{curl}\left(\boldsymbol{H}_{0}\right)=\boldsymbol{j} .
$$

Now, let us assume that $\Omega$ is occupied by a ferromagnetic material with relative magnetic permeability $\mu_{r}$ given as a function of the Euclidean norm of the magnetic field $\boldsymbol{H}$. In fact, $\mu_{r}: \mathbb{R}^{+} \rightarrow \mathbb{R}^{+}$is a given mapping that depends on the material occupying $\Omega$. Let us set $s=|\boldsymbol{H}|$. Since the relative magnetic permeability of the vacuum is 1 , we define the function $\bar{\mu}_{r}: \mathbb{R}^{3} \times \mathbb{R}^{+} \rightarrow \mathbb{R}^{+}$by

$$
\bar{\mu}_{r}(\boldsymbol{x}, s)=\mu_{r}(s), \quad \text { if } \boldsymbol{x} \in \Omega,
$$




$$
\bar{\mu}_{r}(\boldsymbol{x}, s)=1, \quad \text { if } \boldsymbol{x} \notin \Omega .
$$

Let $\boldsymbol{H}$ and $\boldsymbol{B}$ be the fields produced by this stationary electric current density $\boldsymbol{j}$, which is assumed to be unchanged in the presence of the ferromagnetic material. Stationary Ampere's law leads to

$$
\operatorname{curl}(\boldsymbol{H})=\boldsymbol{j},
$$

and, together with (3), it follows that

$$
\operatorname{curl}\left(\boldsymbol{H}-\boldsymbol{H}_{0}\right)=0 \quad \text { in } \mathbb{R}^{3} .
$$

From this last relation, we conclude that there exists a scalar field $\psi$ satisfying

$$
\boldsymbol{H}-\boldsymbol{H}_{0}=\boldsymbol{\nabla} \psi .
$$

Taking into account Gauss' law $\operatorname{div}(\boldsymbol{B})=0$ together with the constitutive law $\boldsymbol{B}=\mu_{0} \bar{\mu}_{r} \boldsymbol{H}$ and (6), we obtain

$$
\operatorname{div}\left(\bar{\mu}_{r}\left(\boldsymbol{H}_{0}+\boldsymbol{\nabla} \psi\right)\right)=0 \quad \text { in } \mathbb{R}^{3} .
$$

Since $\boldsymbol{H}_{0}$ is given by (1), it suffices to compute $\psi$ from (7) in order to obtain $\boldsymbol{H}$ from (6). In order to make this problem more precise, let us make the following remarks.

Remark 1. In order to obtain a finite energy, the fields $\boldsymbol{H}$ and $\boldsymbol{H}_{0}$ are of order $\mathcal{O}\left(|\boldsymbol{x}|^{-2}\right)$ when $\boldsymbol{x}$ tends to infinity and we can assume that $\psi(\boldsymbol{x})=\mathcal{O}\left(|\boldsymbol{x}|^{-1}\right)$ when $|\boldsymbol{x}| \rightarrow \infty$.

Remark 2. In the bounded $\Omega$, the relative magnetic permeability $\mu_{r}$ is a function depending on $s=|\boldsymbol{H}|$. By using (6), we can thus write $\mu_{r}=\mu_{r}\left(\left|\boldsymbol{H}_{0}+\boldsymbol{\nabla} \psi\right|\right)$, which implies that the problem (7) is non linear.

However, in $\mathbb{R}^{3}-\bar{\Omega}$, where $\bar{\Omega}$ is the closure of $\Omega$, we have $\bar{\mu}_{r}=1$. Therefore, by using (3) and (7), the function $\psi$ is harmonic outside $\bar{\Omega}$ and the following relation holds:

$$
\Delta \psi=0 \quad \text { in } \mathbb{R}^{3}-\bar{\Omega} .
$$

\section{The mathematical problem}

In order to establish a well-posed mathematical formulation of Problem (7), we formally multiply (7) by a smooth function $\varphi$, which is $\mathcal{O}\left(|\boldsymbol{x}|^{-1}\right)$ when $|\boldsymbol{x}| \rightarrow \infty$, and we integrate it by parts to obtain

$$
\int_{\mathbb{R}^{3}} \bar{\mu}_{r}\left(\cdot,\left|\boldsymbol{H}_{0}+\boldsymbol{\nabla} \psi\right|\right)\left(\boldsymbol{H}_{0}+\boldsymbol{\nabla} \psi\right) \cdot \boldsymbol{\nabla} \varphi d x=0 .
$$

By setting

$$
a_{\psi}(\lambda, \varphi)=\int_{\mathbb{R}^{3}} \bar{\mu}_{r}\left(\boldsymbol{x},\left|\boldsymbol{H}_{0}(\boldsymbol{x})+\nabla \psi(\boldsymbol{x})\right|\right) \nabla \lambda(\boldsymbol{x}) \cdot \boldsymbol{\nabla} \varphi(\boldsymbol{x}) d x
$$

and

$$
L_{\psi}(\varphi)=-\int_{\mathbb{R}^{3}} \bar{\mu}_{r}\left(\boldsymbol{x},\left|\boldsymbol{H}_{0}(\boldsymbol{x})+\nabla \psi(\boldsymbol{x})\right|\right) \boldsymbol{H}_{0}(\boldsymbol{x}) \cdot \nabla \varphi(\boldsymbol{x}) d x
$$

we are actually looking for $\psi$ satisfying

$$
a_{\psi}(\psi, \varphi)=L_{\psi}(\varphi) \text { for every } \varphi=\mathcal{O}\left(|\boldsymbol{x}|^{-1}\right) \text { when }|\boldsymbol{x}| \rightarrow \infty .
$$

Now, in order to specify this problem, we define the Beppo-Levi space of order 1 (see [5, Chapter IX]) by

$$
W^{1}\left(\mathbb{R}^{3}\right)=\left\{\varphi: \mathbb{R}^{3} \rightarrow \mathbb{R} \text { s.t. } \frac{\varphi(\boldsymbol{x})}{1+|\boldsymbol{x}|} \in L^{2}\left(\mathbb{R}^{3}\right) \text { and } \boldsymbol{\nabla} \varphi \in L^{2}\left(\mathbb{R}^{3}\right)\right\},
$$


where $L^{2}\left(\mathbb{R}^{3}\right)$ is the space of all Lesbegue square integrable functions on $\mathbb{R}^{3}$. The semi-norm $|\varphi|_{W^{1}\left(\mathbb{R}^{3}\right)}=$ $\|\nabla \varphi\|_{L^{2}\left(\mathbb{R}^{3}\right)}$ is a norm on $W^{1}\left(\mathbb{R}^{3}\right)$.

With these definitions and notations, the weak form of Problem (7) reads as follows:

Find $\psi \in W^{1}\left(\mathbb{R}^{3}\right)$ such that

$$
a_{\psi}(\psi, \varphi)=L_{\psi}(\varphi), \quad \forall \varphi \in W^{1}\left(\mathbb{R}^{3}\right)
$$

Remark 3. The field $\boldsymbol{H}_{0}$ is given by the relation (1). In practice, the support of the current density $\boldsymbol{j}$ is compact in $\mathbb{R}^{3}$ and its measure is not vanishing. Hence $\boldsymbol{H}_{0}=\mathcal{O}\left(|\boldsymbol{x}|^{-2}\right)$ when $|\boldsymbol{x}| \rightarrow \infty$ and $\boldsymbol{H}_{0} \in L^{2}\left(\mathbb{R}^{3}\right)$. Problem (9) is therefore formulated in the appropriate spaces.

Remark 4. Since $\bar{\mu}_{r}=1$ outside $\bar{\Omega}$ and $\operatorname{div} \boldsymbol{H}_{0}=0$ in $\mathbb{R}^{3}$ (see (3) with $\boldsymbol{B}_{0}=\mu_{0} \boldsymbol{H}_{0}$ ), we obtain for all $\varphi \in W^{1}\left(\mathbb{R}^{3}\right)$ :

$$
\begin{aligned}
L_{\psi}(\varphi) & =-\int_{\mathbb{R}^{3}} \bar{\mu}_{r}\left(\cdot,\left|\boldsymbol{H}_{0}+\boldsymbol{\nabla} \psi\right|\right) \boldsymbol{H}_{0} \cdot \boldsymbol{\nabla} \varphi d x \\
& =-\int_{\Omega} \mu_{r}\left(\left|\boldsymbol{H}_{0}+\boldsymbol{\nabla} \psi\right|\right) \boldsymbol{H}_{0} \cdot \boldsymbol{\nabla} \varphi d x-\int_{\mathbb{R}^{3}-\Omega} \boldsymbol{H}_{0} \cdot \boldsymbol{\nabla} \varphi d x \\
& =-\int_{\Omega}\left[\mu_{r}\left(\left|\boldsymbol{H}_{0}+\boldsymbol{\nabla} \psi\right|\right)-1\right] \boldsymbol{H}_{0} \cdot \boldsymbol{\nabla} \varphi d x-\int_{\mathbb{R}^{3}} \boldsymbol{H}_{0} \cdot \boldsymbol{\nabla} \varphi d x \\
& =-\int_{\Omega}\left[\mu_{r}\left(\left|\boldsymbol{H}_{0}+\boldsymbol{\nabla} \psi\right|\right)-1\right] \boldsymbol{H}_{0} \cdot \boldsymbol{\nabla} \varphi d x-\int_{\mathbb{R}^{3}} \operatorname{div} \boldsymbol{H}_{0} \varphi d x \\
& =-\int_{\Omega}\left[\mu_{r}\left(\left|\boldsymbol{H}_{0}+\boldsymbol{\nabla} \psi\right|\right)-1\right] \boldsymbol{H}_{0} \cdot \boldsymbol{\nabla} \varphi d x .
\end{aligned}
$$

This relation shows that we have to compute $\boldsymbol{H}_{0}$ only in $\Omega$ when evaluating $L_{\psi}(\varphi)$. A similar remark holds for the computation of $a_{\psi}(\lambda, \varphi)$. Problem (9) is thus equivalent to finding $\psi \in W^{1}\left(\mathbb{R}^{3}\right)$ such that

$$
a_{\psi}(\psi, \varphi)=-\int_{\Omega}\left[\mu_{r}\left(\left|\boldsymbol{H}_{0}+\nabla \psi\right|\right)-1\right] \boldsymbol{H}_{0} \cdot \nabla \varphi d x, \quad \forall \varphi \in W^{1}\left(\mathbb{R}^{3}\right) .
$$

As a consequence, when $\mu_{r}=1$ in $\Omega$, that is to say without ferromagnetism effect, we have $a_{\psi}(\psi, \varphi)=$ $\int_{\mathbb{R}^{3}} \boldsymbol{\nabla} \psi \cdot \boldsymbol{\nabla} \varphi d x=0$ for all $\varphi \in W^{1}\left(\mathbb{R}^{3}\right)$, which implies $\psi=0$ and so $\boldsymbol{H}=\boldsymbol{H}_{0}$.

Now, let us assume that the function $\mu_{r}: \mathbb{R}^{+} \rightarrow \mathbb{R}^{+}$satisfies the following properties:

(H1) $\mu_{r}$ is a $C^{1}$ decreasing function on $[0, \infty)$ satisfying:

1) $0<\beta^{\prime} \leqslant \mu_{r}(s) \leqslant \beta$,

2) $\mu_{r}(s)+s \mu_{r}^{\prime}(s) \geqslant \alpha>0$,

for all $s \in \mathbb{R}^{+}$, where $\beta, \beta^{\prime}$ and $\alpha$ are positive constants. Let us mention that (H1) is satisfied in practice with $\beta^{\prime}=\alpha=1$.

Remark 5. The hypothesis 2) above means that $\frac{d}{d s}\left(s \mu_{r}(s)\right) \geqslant \alpha$ and consequently, if $g(s)$ is a primitive of $s \mu_{r}(s)$, then the function $g$ is strictly convex. From a physical point of view, $g(|\boldsymbol{H}|)$ is proportional to the magnetic energy density, which is assumed to be convex by Hypothesis 2).

Theorem 1. Under Hypothesis (H1) and when $\boldsymbol{H}_{0} \in L^{2}\left(\mathbb{R}^{3}\right)$, Problem (9) admits a unique solution $\psi \in W^{1}\left(\mathbb{R}^{3}\right)$.

Proof. Let us define the function $g: \mathbb{R}^{+} \rightarrow \mathbb{R}^{+}$by $g(s)=\int_{0}^{s} t \mu_{r}(t) d t$ for $s \geqslant 0$, and the functional $\mathcal{L}: W^{1}\left(\mathbb{R}^{3}\right) \rightarrow \mathbb{R}$ by

$$
\mathcal{L}(\varphi)=\int_{\Omega} g\left(\left|\boldsymbol{H}_{0}+\boldsymbol{\nabla} \varphi\right|\right) d x+\frac{1}{2} \int_{\mathbb{R}^{3}-\Omega}\left|\boldsymbol{H}_{0}+\nabla \varphi\right|^{2} d x
$$

We are going to prove that $\mathcal{L}$ is well-defined, continuous on $W^{1}\left(\mathbb{R}^{3}\right)$, strictly convex and coercive. Then we will conclude that $\mathcal{L}$ possesses a unique minimum $\psi \in W^{1}\left(\mathbb{R}^{3}\right)$, i.e., such that $\mathcal{L}(\psi) \leqslant \mathcal{L}(\varphi)$ for 
all $\varphi \in W^{1}\left(\mathbb{R}^{3}\right)$. Moreover, by using the fact that the gradient of the function $\boldsymbol{\xi} \in \mathbb{R}^{3} \rightarrow|\boldsymbol{\xi}| \in \mathbb{R}$ is given by $\boldsymbol{\xi} /|\boldsymbol{\xi}|$ and by computing the Gâteaux derivative of $\mathcal{L}$ at $\psi$ in the direction $\varphi$, we obtain

$$
\left\langle\mathcal{L}^{\prime}(\psi) ; \varphi\right\rangle=\int_{\mathbb{R}^{3}} \bar{\mu}_{r}\left(\boldsymbol{x},\left|\boldsymbol{H}_{0}(\boldsymbol{x})+\boldsymbol{\nabla} \psi(\boldsymbol{x})\right|\right)\left(\boldsymbol{H}_{0}(\boldsymbol{x})+\boldsymbol{\nabla} \psi(\boldsymbol{x})\right) \cdot \boldsymbol{\nabla} \varphi(\boldsymbol{x}) d x,
$$

which implies that $\psi$ is the unique solution to Problem (9).

1) We start with the continuity of $\mathcal{L}$. Hypothesis (H1) implies that $0 \leqslant g(s) \leqslant \frac{1}{2} \beta s^{2}$ for all $s \geqslant 0$ and $\mathcal{L}(\varphi) \leqslant \frac{1}{2} \beta\left\|\boldsymbol{H}_{0}+\boldsymbol{\nabla} \varphi\right\|_{L^{2}\left(\mathbb{R}^{3}\right)}^{2}$ which shows that $\mathcal{L}$ is well defined on $W^{1}\left(\mathbb{R}^{3}\right)$. Moreover, we have $\frac{d}{d s} g(s)=s \mu_{r}(s) \leqslant \beta s$ and we easily prove that $\mathcal{L}$ is continuous on $W^{1}\left(\mathbb{R}^{3}\right)$.

2 ) In order to prove that $\mathcal{L}$ is a strictly convex functional, we use again Hypotheses (H1). We have $g^{\prime}(s)=s \mu_{r}(s) \geqslant \beta^{\prime} s$ and finally $g^{\prime \prime}(s)=\mu_{r}(s)+s \mu_{r}^{\prime}(s) \geqslant \alpha$ for all $s \in \mathbb{R}^{+}$. It follows that the function $g: \mathbb{R}^{+} \rightarrow \mathbb{R}^{+}$is strictly increasing and strictly convex, and from these two last properties, that the mapping $\boldsymbol{y} \in \mathbb{R}^{3} \rightarrow g(|\boldsymbol{y}|)$ is strictly convex.

Now, if $\varphi, \omega \in W^{1}\left(\mathbb{R}^{3}\right)$ with $\varphi \neq \omega$, we have $\nabla \varphi \neq \nabla \omega$. Let $\Lambda \subset \mathbb{R}^{3}$ be defined by $\Lambda=$ $\left\{\boldsymbol{x} \in \mathbb{R}^{3}: \boldsymbol{\nabla} \varphi(\boldsymbol{x}) \neq \boldsymbol{\nabla} \omega(\boldsymbol{x})\right.$ a.e. $\}$. If $0<\lambda<1$, and if meas $(\Lambda \cap \Omega) \neq 0$, we have

$$
\begin{aligned}
\int_{\Omega} g\left(\left|\boldsymbol{H}_{0}+\lambda \boldsymbol{\nabla} \varphi+(1-\lambda) \boldsymbol{\nabla} \omega\right|\right) d x \\
\quad=\int_{\Omega} g\left(\left|\lambda\left(\boldsymbol{H}_{0}+\boldsymbol{\nabla} \varphi\right)+(1-\lambda)\left(\boldsymbol{H}_{0}+\boldsymbol{\nabla} \omega\right)\right|\right) d x \\
\quad<\lambda \int_{\Omega} g\left(\left|\boldsymbol{H}_{0}+\boldsymbol{\nabla} \varphi\right|\right) d x+(1-\lambda) \int_{\Omega} g\left(\left|\boldsymbol{H}_{0}+\boldsymbol{\nabla} \omega\right|\right) d x .
\end{aligned}
$$

If meas $\left(\Lambda \cap\left(\mathbb{R}^{3}-\Omega\right)\right) \neq 0$, we have

$$
\begin{aligned}
& \int_{\mathbb{R}^{3}-\Omega}\left|\boldsymbol{H}_{0}+\lambda \boldsymbol{\nabla} \varphi+(1-\lambda) \boldsymbol{\nabla} \omega\right|^{2} d x \\
& \quad<\lambda \int_{\mathbb{R}^{3}-\Omega}\left|\boldsymbol{H}_{0}+\boldsymbol{\nabla} \varphi\right|^{2} d x+(1-\lambda) \int_{\mathbb{R}^{3}-\Omega}\left|\boldsymbol{H}_{0}+\boldsymbol{\nabla} \omega\right|^{2} d x .
\end{aligned}
$$

Finally, since

$$
\mathcal{L}(\varphi)=\int_{\Omega} g\left(\left|\boldsymbol{H}_{0}+\boldsymbol{\nabla} \varphi\right|\right) d x+\frac{1}{2} \int_{\mathbb{R}^{3}-\Omega}\left|\boldsymbol{H}_{0}+\nabla \varphi\right|^{2} d x
$$

we know that $\mathcal{L}$ is strictly convex.

3) It remains to prove that $\mathcal{L}$ is coercive, i.e., there exist two constants $\zeta>0$ and $\eta \in \mathbb{R}$ satisfying $\mathcal{L}(\varphi) \geqslant \zeta\|\varphi\|_{W^{1}\left(\mathbb{R}^{3}\right)}+\eta$. By using Hypothesis $(\mathrm{H} 1)$, we verify that $g(s) \geqslant \frac{1}{2} \beta^{\prime} s^{2}$ for all $s \in \mathbb{R}^{+}$. By setting $\zeta=\frac{1}{2} \min \left(\beta^{\prime}, 1\right)$, it follows that

$$
\mathcal{L}(\varphi) \geqslant \zeta \int_{\mathbb{R}^{3}}\left|\boldsymbol{H}_{0}+\boldsymbol{\nabla} \varphi\right|^{2} d x \geqslant \zeta \int_{\mathbb{R}^{3}}\left(\left|\boldsymbol{H}_{0}\right|^{2}+2 \boldsymbol{H}_{0} \cdot \boldsymbol{\nabla} \varphi+|\boldsymbol{\nabla} \varphi|^{2}\right) d x \geqslant \zeta\left(|\varphi|_{W^{1}\left(\mathbb{R}^{3}\right)}-\widetilde{\eta}\right)
$$

with $\widetilde{\eta}=\left\|\boldsymbol{H}_{0}\right\|_{L^{2}\left(\mathbb{R}^{3}\right)}+1$.

From the above results, it follows that $\mathcal{L}$ is weakly lower semicontinuous (see [4, Chapter 3, Theorem 1.2]) and since $W^{1}\left(\mathbb{R}^{3}\right)$ is a Hilbert space (consequently reflexive), there exists $\psi \in W^{1}\left(\mathbb{R}^{3}\right)$ satisfying $\mathcal{L}(\psi) \leqslant \mathcal{L}(\varphi)$ for all $\varphi \in W^{1}\left(\mathbb{R}^{3}\right)$ (see [4, Chapter 3, Theorem 1.1]). Moreover, the strict convexity of $\mathcal{L}$ implies the uniqueness of the minimum. This minimum is the unique solution to the following equation involving the Gâteaux derivative of $\mathcal{L}$ :

$$
\left\langle\mathcal{L}^{\prime}(\psi) ; \theta\right\rangle=\int_{\mathbb{R}^{3}} \bar{\mu}_{r}\left(\cdot,\left|\boldsymbol{H}_{0}+\nabla \psi\right|\right)\left(\boldsymbol{H}_{0}+\nabla \psi\right) \cdot \nabla \theta d x=0
$$

for all $\theta \in W^{1}\left(\mathbb{R}^{3}\right)$ (see [4, Chapter 3, Theorem 4.4]). Therefore, $\psi$ is the solution to Problem (9). 


\section{An iterative method converging to $\psi \in W^{1}\left(\mathbb{R}^{3}\right)$}

As mentioned earlier, in order to compute $\boldsymbol{H}$, it suffices to know how to compute the solution $\psi$ to Problem (9), as the magnetic field is given by $\boldsymbol{H}=\boldsymbol{H}_{0}+\nabla \psi$.

Let us consider the mapping $F: W^{1}\left(\mathbb{R}^{3}\right) \rightarrow W^{1}\left(\mathbb{R}^{3}\right)$ defined by

$$
\int_{\mathbb{R}^{3}} \bar{\mu}_{r}\left(\cdot,\left|\boldsymbol{H}_{0}+\nabla z\right|\right)\left(\boldsymbol{H}_{0}+\nabla F(z)\right) \cdot \nabla \varphi d x=0 \quad \text { for all } \varphi, z \in W^{1}\left(\mathbb{R}^{3}\right) .
$$

Clearly, $F$ is well defined since Hypothesis (H1) implies that the bilinear form $a_{z}(\cdot, \cdot)$ is continuous and coercive on $W^{1}\left(\mathbb{R}^{3}\right)$ for every $z \in W^{1}\left(\mathbb{R}^{3}\right)$. Moreover, we have

$$
\|\boldsymbol{\nabla} F(z)\|_{L^{2}\left(\mathbb{R}^{3}\right)} \leqslant \beta\left\|\boldsymbol{H}_{0}\right\|_{L^{2}\left(\mathbb{R}^{3}\right)}, \quad \forall z \in W^{1}\left(\mathbb{R}^{3}\right) .
$$

We observe that $\psi$ is the solution to Problem (9) if and only if $\psi$ is a fixed point of $F$ in $W^{1}\left(\mathbb{R}^{3}\right)$, i.e.,

$$
\psi \in W^{1}\left(\mathbb{R}^{3}\right) \text { satisfies } \psi=F(\psi) \text {. }
$$

Remark 6. Looking at the definition of $\bar{\mu}_{r}$, it is easy to see that the value of the function $F(z)$ actually depends only on the restriction of $z$ on the bounded domain $\Omega$. As a consequence, if $G(z)$ is the restriction of $F(z)$ on $\Omega$, then $\psi$ is the solution to Problem (9) if and only if $\psi$ is a fixed point of $G$ in the classical Sobolev space $H^{1}(\Omega)$.

In order to compute the solution $\psi$ to Problem (9), we will use the following relaxed fixed point method:

a) Set $\psi_{0}$ in $W^{1}\left(\mathbb{R}^{3}\right)$ arbitrarily and choose $\epsilon>0$.

b) Compute, for $k=0,1,2, \ldots$ :

$$
\begin{aligned}
\widetilde{\psi}_{k+1} & =F\left(\psi_{k}\right), \\
\psi_{k+1} & =(1-\epsilon) \psi_{k}+\epsilon \widetilde{\psi}_{k+1} .
\end{aligned}
$$

Remark 7. The solution to the equation $\widetilde{\psi}_{k+1}=F\left(\psi_{k}\right)$ is equivalent to the solution, at each step, to the linear problem: for given $\psi_{k}$, find $\widetilde{\psi}_{k+1} \in W^{1}\left(\mathbb{R}^{3}\right)$ satisfying:

$$
a_{\psi_{k}}\left(\widetilde{\psi}_{k+1}, \varphi\right)=L_{\psi_{k}}(\varphi), \quad \forall \varphi \in W^{1}\left(\mathbb{R}^{3}\right), k=0,1,2, \ldots
$$

Before proving the convergence of $\psi_{k}$ to the solution $\psi$ to Problem (9), we establish two lemmas which are variations of the results found in [2].

Lemma 1. Under Hypothesis (H1) we have

$$
\left(\mu_{r}(|\boldsymbol{\xi}|) \boldsymbol{\xi}-\mu_{r}(|\boldsymbol{\eta}|) \boldsymbol{\eta}\right) \cdot(\boldsymbol{\xi}-\boldsymbol{\eta}) \geqslant \min \left(\alpha, \beta^{\prime}\right)|\boldsymbol{\xi}-\boldsymbol{\eta}|^{2} \quad \text { for all } \boldsymbol{\xi}, \boldsymbol{\eta} \in \mathbb{R}^{3} \text {. }
$$

Proof. First, we observe that Hypothesis (H1) implies that $\frac{d}{d s}\left(s \mu_{r}(s)\right) \geqslant \alpha$ for $s \geqslant 0$ and by integrating this inequality between $r$ and $t$ with $0 \leqslant r \leqslant t$, we obtain

$$
t \mu_{r}(t)-r \mu_{r}(r) \geqslant \alpha(t-r)
$$

Now, if $\boldsymbol{\xi}, \boldsymbol{\eta} \in \mathbb{R}^{3}$, we obtain

$$
\begin{aligned}
S: & =\left(\mu_{r}(|\boldsymbol{\xi}|) \boldsymbol{\xi}-\mu_{r}(|\boldsymbol{\eta}|) \boldsymbol{\eta}\right) \cdot(\boldsymbol{\xi}-\boldsymbol{\eta}) \\
& =\mu_{r}(|\boldsymbol{\xi}|)|\boldsymbol{\xi}|^{2}+\mu_{r}(|\boldsymbol{\eta}|)|\boldsymbol{\eta}|^{2}-\left(\mu_{r}(|\boldsymbol{\xi}|)+\mu_{r}(|\boldsymbol{\eta}|)\right) \cdot(\boldsymbol{\xi} \cdot \boldsymbol{\eta}) \\
& =\left(\mu_{r}(|\boldsymbol{\xi}|)|\boldsymbol{\xi}|-\mu_{r}(|\boldsymbol{\eta}|)|\boldsymbol{\eta}|\right)(|\boldsymbol{\xi}|-|\boldsymbol{\eta}|)+\left(\mu_{r}(|\boldsymbol{\xi}|)+\mu_{r}(|\boldsymbol{\eta}|)\right) \cdot(|\boldsymbol{\xi}||\boldsymbol{\eta}|-\boldsymbol{\xi} \cdot \boldsymbol{\eta}) .
\end{aligned}
$$

By using the relation (14), we obtain

$$
S \geqslant \alpha(|\boldsymbol{\xi}|-|\boldsymbol{\eta}|)^{2}+\left(\mu_{r}(|\boldsymbol{\xi}|)+\mu_{r}(|\boldsymbol{\eta}|)\right) \cdot(|\boldsymbol{\xi}||\boldsymbol{\eta}|-\boldsymbol{\xi} \cdot \boldsymbol{\eta}) .
$$


Since $\beta^{\prime} \leqslant \mu_{r}(s)$ for all $s \in \mathbb{R}$ and $\boldsymbol{\xi} \cdot \boldsymbol{\eta} \leqslant|\boldsymbol{\xi}||\boldsymbol{\eta}|$, we finally have

$$
S \geqslant \alpha(|\boldsymbol{\xi}|-|\boldsymbol{\eta}|)^{2}+2 \beta^{\prime}(|\boldsymbol{\xi}||\boldsymbol{\eta}|-\boldsymbol{\xi} . \boldsymbol{\eta}) \geqslant \min \left(\alpha, \beta^{\prime}\right)|\boldsymbol{\xi}-\boldsymbol{\eta}|^{2} .
$$

The proof is complete.

Lemma 2. Under Hypothesis (H1) we have

$$
\left|\mu_{r}(|\boldsymbol{\xi}|) \boldsymbol{\xi}-\mu_{r}(|\boldsymbol{\eta}|) \boldsymbol{\eta}\right| \leqslant \beta|\boldsymbol{\xi}-\boldsymbol{\eta}| \quad \text { for all } \boldsymbol{\xi}, \boldsymbol{\eta} \in \mathbb{R}^{3} .
$$

Proof. If $0 \leqslant r \leqslant t$, we have

$$
t \mu_{r}(t)-r \mu_{r}(r)=\int_{r}^{t}\left(s \mu_{r}^{\prime}(s)+\mu_{r}(s)\right) d s .
$$

By using Hypothesis (H1), we see that the mapping $s \rightarrow s \mu_{r}(s)$ is increasing and since $\mu_{r}^{\prime}(s) \leqslant 0$, we obtain

$$
0 \leqslant t \mu_{r}(t)-r \mu_{r}(r) \leqslant \sup _{s \geqslant 0} \mu_{r}(s)(t-r) .
$$

In conclusion, we have for every $t, r \geqslant 0$,

$$
\left|t \mu_{r}(t)-r \mu_{r}(r)\right| \leqslant \beta|t-r| .
$$

Let us take $\boldsymbol{\xi}, \boldsymbol{\eta} \in \mathbb{R}^{3}$ and compute

$$
\begin{aligned}
\mid \mu_{r} & (|\boldsymbol{\xi}|) \boldsymbol{\xi}-\left.\mu_{r}(|\boldsymbol{\eta}|) \boldsymbol{\eta}\right|^{2} \\
& =\mu_{r}^{2}(|\boldsymbol{\xi}|)|\boldsymbol{\xi}|^{2}+\mu_{r}^{2}(|\boldsymbol{\eta}|)|\boldsymbol{\eta}|^{2}-2 \mu_{r}(|\boldsymbol{\xi}|) \mu_{r}(|\boldsymbol{\eta}|)(\boldsymbol{\xi} \cdot \boldsymbol{\eta}) \\
& =\left(\mu_{r}(|\boldsymbol{\xi}|)|\boldsymbol{\xi}|-\mu_{r}(|\boldsymbol{\eta}|)|\boldsymbol{\eta}|\right)^{2}+2\left(\mu_{r}(|\boldsymbol{\xi}|) \mu_{r}(|\boldsymbol{\eta}|)\right)(|\boldsymbol{\xi}||\boldsymbol{\eta}|-\boldsymbol{\xi} \cdot \boldsymbol{\eta}) \\
& \leqslant \beta^{2}(|\boldsymbol{\xi}|-|\boldsymbol{\eta}|)^{2}+2 \beta^{2}(|\boldsymbol{\xi}||\boldsymbol{\eta}|-\boldsymbol{\xi} \cdot \boldsymbol{\eta}) \\
& \leqslant \beta^{2}\left[(|\boldsymbol{\xi}|-|\boldsymbol{\eta}|)^{2}+2(|\boldsymbol{\xi}||\boldsymbol{\eta}|-\boldsymbol{\xi} \cdot \boldsymbol{\eta})\right]=\beta^{2}|\boldsymbol{\xi}-\boldsymbol{\eta}|^{2} .
\end{aligned}
$$

The proof is complete.

Theorem 2. Assume that Hypothesis (H1) is satisfied and $\boldsymbol{H}_{0} \in L^{2}\left(\mathbb{R}^{3}\right)$. Then if $\epsilon<2 / \beta$, then the algorithms (11), (12) converges. The limit solution to the iterative algorithm is the function solution $\psi$ to Problem (9) and

$$
\lim _{k \rightarrow \infty}\left\|\boldsymbol{\nabla}\left(\psi_{k}-\psi\right)\right\|_{L^{2}\left(\mathbb{R}^{3}\right)}=0
$$

Proof. Let us define $\mu_{k}=\bar{\mu}_{r}\left(\cdot,\left|\boldsymbol{H}_{0}+\boldsymbol{\nabla} \psi_{k}\right|\right)$. It follows that $\mu_{k}$ is a function of $\boldsymbol{x} \in \mathbb{R}^{3}$ with $\beta^{\prime} \leqslant \mu_{k} \leqslant$ $\beta$, and if $\kappa_{k} \in W^{1}\left(\mathbb{R}^{3}\right)$ is such that

$$
\int_{\mathbb{R}^{3}} \mu_{k} \boldsymbol{\nabla} \kappa_{k} \cdot \boldsymbol{\nabla} \varphi d x=\int_{\mathbb{R}^{3}} \mu_{k}\left(\boldsymbol{H}_{0}+\boldsymbol{\nabla} \psi_{k}\right) \cdot \boldsymbol{\nabla} \varphi d x \text { for all } \varphi \in W^{1}\left(\mathbb{R}^{3}\right),
$$

then we have $\kappa_{k}=\psi_{k}-F\left(\psi_{k}\right)$.

Now, we define $q(\tau)=-\mathcal{L}\left(\psi_{k}-\tau \kappa_{k}\right)$. By using (10) we have

$$
\begin{aligned}
\frac{d}{d \tau} q(\tau) & =\left\langle\mathcal{L}^{\prime}\left(\psi_{k}-\tau \kappa_{k}\right) ; \kappa_{k}\right\rangle \\
& =\int_{\mathbb{R}^{3}} \bar{\mu}_{r}\left(\cdot,\left|\boldsymbol{H}_{0}+\boldsymbol{\nabla}\left(\psi_{k}-\tau \kappa_{k}\right)\right|\right)\left(\boldsymbol{H}_{0}+\boldsymbol{\nabla}\left(\psi_{k}-\tau \kappa_{k}\right)\right) \cdot \boldsymbol{\nabla} \kappa_{k} d x \\
& =\int_{\mathbb{R}^{3}}\left[\bar{\mu}_{r}\left(\cdot,\left|\boldsymbol{H}_{0}+\boldsymbol{\nabla}\left(\psi_{k}-\tau \kappa_{k}\right)\right|\right)\left(\boldsymbol{H}_{0}+\boldsymbol{\nabla}\left(\psi_{k}-\tau \kappa_{k}\right)\right)\right.
\end{aligned}
$$




$$
\left.-\mu_{k}\left(\boldsymbol{H}_{0}+\nabla \psi_{k}\right)\right] \cdot \nabla \kappa_{k} d x+\int_{\mathbb{R}^{3}} \mu_{k}\left|\nabla \kappa_{k}\right|^{2} d x .
$$

By using Lemma 2 we obtain

$$
\frac{d}{d \tau} q(\tau) \geqslant \int_{\mathbb{R}^{3}} \mu_{k}\left|\nabla \kappa_{k}\right|^{2} d x-\beta \int_{\mathbb{R}^{3}}|\tau|\left|\nabla \kappa_{k}\right|^{2} d x \geqslant(1-\beta|\tau|) \int_{\mathbb{R}^{3}}\left|\nabla \kappa_{k}\right|^{2} d x .
$$

It follows that for $t \geqslant 0$ we have

$$
\mathcal{L}\left(\psi_{k}-t \kappa_{k}\right)-\mathcal{L}\left(\psi_{k}\right)=-\int_{0}^{t} \frac{d}{d \tau} q(\tau) d \tau \leqslant\left(\beta \frac{t^{2}}{2}-t\right) \int_{\mathbb{R}^{3}}\left|\nabla \kappa_{k}\right|^{2} d x .
$$

By taking $t=\epsilon$ and since $\kappa_{k}=\psi_{k}-F\left(\psi_{k}\right)$, the relations (11) and (12) imply

$$
\mathcal{L}\left(\psi_{k+1}\right)-\mathcal{L}\left(\psi_{k}\right) \leqslant\left(\beta \frac{\epsilon^{2}}{2}-\epsilon\right) \int_{\mathbb{R}^{3}}\left|\nabla \kappa_{k}\right|^{2} d x,
$$

and when $\epsilon<\frac{2}{\beta}$, the sequence $\left(\mathcal{L}\left(\psi_{k}\right)\right)_{k=0}^{\infty}$ is decreasing and bounded from below by $\mathcal{L}(\psi)$. Thus this sequence is converging and we have

$$
\lim _{k \rightarrow \infty}\left\|\nabla \kappa_{k}\right\|_{L^{2}\left(R^{3}\right)}=0 .
$$

Now we use Lemma 1 to obtain, when $\gamma=\min \left(\alpha, \beta^{\prime}, 1\right)$ :

$$
\begin{aligned}
\gamma \int_{\mathbb{R}^{3}}\left|\boldsymbol{\nabla}\left(\psi-\psi_{k}\right)\right|^{2} d x \leqslant & \int_{\mathbb{R}^{3}}\left[\bar{\mu}_{r}\left(\left|\boldsymbol{H}_{0}+\boldsymbol{\nabla} \psi\right|\right)\left(\boldsymbol{H}_{0}+\boldsymbol{\nabla} \psi\right)\right. \\
& \left.-\bar{\mu}_{r}\left(\left|\boldsymbol{H}_{0}+\boldsymbol{\nabla} \psi_{k}\right|\right)\left(\boldsymbol{H}_{0}+\boldsymbol{\nabla} \psi_{k}\right)\right] \cdot \boldsymbol{\nabla}\left(\psi-\psi_{k}\right) d x \\
= & -\int_{\mathbb{R}^{3}} \bar{\mu}_{r}\left(\left|\boldsymbol{H}_{0}+\boldsymbol{\nabla} \psi_{k}\right|\right)\left(\boldsymbol{H}_{0}+\boldsymbol{\nabla} \psi_{k}\right) \cdot \boldsymbol{\nabla}\left(\psi-\psi_{k}\right) d x \\
= & -\int_{\mathbb{R}^{3}} \mu_{k}\left(\boldsymbol{H}_{0}+\boldsymbol{\nabla} \psi_{k}\right) \cdot \boldsymbol{\nabla}\left(\psi-\psi_{k}\right) d x \\
= & -\int_{\mathbb{R}^{3}} \mu_{k} \boldsymbol{\nabla} \kappa_{k} \cdot \boldsymbol{\nabla}\left(\psi-\psi_{k}\right) d x \\
\leqslant & \beta\left\|\kappa_{k}\right\|_{L^{2}\left(\mathbb{R}^{3}\right)}\left\|\boldsymbol{\nabla}\left(\psi-\psi_{k}\right)\right\|_{L^{2}\left(\mathbb{R}^{3}\right)} .
\end{aligned}
$$

Finally, we obtain $\left\|\boldsymbol{\nabla}\left(\psi-\psi_{k}\right)\right\|_{L^{2}\left(\mathbb{R}^{3}\right)} \leqslant \frac{\beta}{\gamma}\left\|\nabla \kappa_{k}\right\|_{L^{2}\left(\mathbb{R}^{3}\right)}$ and consequently, since $\lim _{k \rightarrow \infty}\left\|\nabla \kappa_{k}\right\|_{L^{2}\left(\mathbb{R}^{3}\right)}=0$, we obtain the convergence of $\psi_{k}$ to $\psi$ when $k \rightarrow 0$.

\section{A domain decomposition method}

In the algorithm described in Section 4, we need to solve (13) to compute the iterate $\psi_{k+1}$ from the previous iterate $\psi_{k}$, i.e., we need to find $\widetilde{\psi}_{k+1} \in W^{1}\left(\mathbb{R}^{3}\right)$ satisfying for every $\varphi \in W^{1}\left(\mathbb{R}^{3}\right)$ :

$$
\int_{\mathbb{R}^{3}} \mu_{k} \boldsymbol{\nabla} \widetilde{\psi}_{k+1} \cdot \nabla \varphi d x=-\int_{\mathbb{R}^{3}} \mu_{k} \boldsymbol{H}_{0} \cdot \boldsymbol{\nabla} \varphi d x
$$

where $\mu_{k}=\bar{\mu}_{r}\left(\cdot,\left|\boldsymbol{H}_{0}+\boldsymbol{\nabla} \psi_{k}\right|\right)$.

Following Remark 4 , we obtain for every $\varphi \in W^{1}\left(\mathbb{R}^{3}\right)$ :

$$
\int_{\mathbb{R}^{3}} \mu_{k} \boldsymbol{\nabla} \widetilde{\psi}_{k+1} \cdot \nabla \varphi d x=\int_{\Omega}\left[\mu_{k}-1\right] \boldsymbol{H}_{0} \cdot \nabla \varphi d x .
$$

Since $\mu_{k}=1$ outside $\Omega$, Equation (15) implies that $\widetilde{\psi}_{k+1}$ is harmonic outside $\Omega$. 
Let us consider an open ball $B_{r}$ containing $\bar{\Omega}$, centered at the origin, with radius $r$, and with boundary $\partial B_{r}$. The Poisson formula [8] reads:

$$
\widetilde{\psi}_{k+1}(\boldsymbol{x})=\frac{\left(|\boldsymbol{x}|^{2}-r^{2}\right)}{4 \pi r} \int_{\partial B_{r}} \frac{\widetilde{\psi}_{k+1}(\boldsymbol{y})}{|\boldsymbol{x}-\boldsymbol{y}|^{3}} d s(y), \quad \forall \boldsymbol{x} \in \mathbb{R}^{3}-B_{r} .
$$

Therefore, if $B_{R}$ is a ball centered at the origin and with radius $R>r$, Problem (15) is equivalent to finding $\widetilde{\psi}_{k+1} \in H^{1}\left(B_{R}\right)$ satisfying (16) on the boundary $\partial B_{R}$ of $B_{R}$, and for every $\varphi \in H_{0}^{1}\left(B_{R}\right)$ :

$$
\int_{B_{R}} \mu_{k} \boldsymbol{\nabla} \widetilde{\psi}_{k+1} \cdot \nabla \varphi d x=\int_{\Omega}\left[\mu_{k}-1\right] \boldsymbol{H}_{0} \cdot \nabla \varphi d x
$$

Here $H^{1}\left(B_{R}\right)=\left\{\varphi \in L^{2}\left(B_{R}\right)\right.$ with $\left.|\nabla \varphi| \in L^{2}\left(B_{R}\right)\right\}$ is the classical Sobolev space and $H_{0}^{1}\left(B_{R}\right)$ is the set of functions in $H^{1}\left(B_{R}\right)$ with trace vanishing on the boundary $\partial B_{R}$.

In order to compute the solution $\widetilde{\psi}_{k+1}$ to Problem (17), we use a Schwarz algorithm with overlap and Dirichlet-Dirichlet boundary conditions. The two domains are $B_{R}$ and $\mathbb{R}^{3}-\bar{B}_{r}$, their overlap being $B_{R}-\bar{B}_{r}$. In order to solve the harmonic problem in $\mathbb{R}^{3}-\bar{B}_{r}$, we use Poisson's formula. The algorithm becomes:

a) Initialize $\varkappa^{(0)} \in H_{0}^{1}\left(B_{R}\right)$ by solving

$$
\int_{B_{R}} \mu_{k} \boldsymbol{\nabla} \varkappa^{(0)} \cdot \nabla \varphi d x=\int_{\Omega}\left[\mu_{k}-1\right] \boldsymbol{H}_{0} \cdot \nabla \varphi d x, \quad \forall \varphi \in H_{0}^{1}\left(B_{R}\right) .
$$

b) For $n=0,1,2,3,4, \ldots$, compute $\varkappa^{(n+1)} \in H^{1}\left(B_{R}\right)$ satisfying

$$
\int_{B_{R}} \mu_{k} \boldsymbol{\nabla} \varkappa^{(n+1)} \cdot \nabla \varphi d x=\int_{\Omega}\left[\mu_{k}-1\right] \boldsymbol{H}_{0} \cdot \nabla \varphi d x, \quad \forall \varphi \in H_{0}^{1}\left(B_{R}\right)
$$

and

$$
\varkappa^{(n+1)}(\boldsymbol{x})=\frac{\left(R^{2}-r^{2}\right)}{4 \pi r} \int_{\partial B_{r}} \frac{\varkappa^{(n)}(\boldsymbol{y})}{|\boldsymbol{x}-\boldsymbol{y}|^{3}} d s(y) \quad \forall \boldsymbol{x} \in \partial B_{R} .
$$

We have the following result:

Theorem 3. If $\widetilde{\psi}_{k+1} \in W^{1}\left(\mathbb{R}^{3}\right)$ is the solution to Problem (15), we have the estimate

$$
\left\|\widetilde{\psi}_{k+1}-\varkappa^{(n)}\right\|_{L^{\infty}\left(B_{R}\right)} \leqslant\left(\frac{r}{R}\right)^{n}\left\|\widetilde{\psi}_{k+1}-\varkappa^{(0)}\right\|_{L^{\infty}\left(\partial B_{R}\right)} .
$$

This estimates implies in particular

$$
\lim _{n \rightarrow \infty}\left\|\widetilde{\psi}_{k+1}-\varkappa^{(n)}\right\|_{L^{\infty}\left(B_{R}\right)}=0 .
$$

Proof. We define $e^{(n)}=\widetilde{\psi}_{k+1}-\varkappa^{(n)}$ on $B_{R}$ and have:

$$
e^{(n)}(\boldsymbol{x})=\frac{\left(R^{2}-r^{2}\right)}{4 \pi r} \int_{\partial B_{r}} \frac{e^{(n-1)}(\boldsymbol{y})}{|\boldsymbol{x}-\boldsymbol{y}|^{3}} d s(\boldsymbol{y}), \quad \forall \boldsymbol{x} \in \partial B_{R} .
$$

It follows that

$$
\left\|e^{(n)}\right\|_{L^{\infty}\left(\partial B_{R}\right)} \leqslant\left\|e^{(n-1)}\right\|_{L^{\infty}\left(\partial B_{r}\right)} \frac{\left(R^{2}-r^{2}\right)}{4 \pi r} \max _{\boldsymbol{x} \in \partial B_{R}} \int_{\partial B_{r}} \frac{1}{|\boldsymbol{x}-\boldsymbol{y}|^{3}} d s(\boldsymbol{y}) .
$$

By considering $\boldsymbol{x}$ at the north pole of the sphere $\partial B_{r}$ and by using the spherical coordinates, we obtain after setting $\bar{\alpha}=r / R$ :

$$
\int_{\partial B_{r}} \frac{1}{|\boldsymbol{x}-\boldsymbol{y}|^{3}} d s(\boldsymbol{y})=\int_{0}^{\pi} d \theta \int_{0}^{2 \pi} d \varphi \frac{r^{2} \sin (\theta)}{\left(r^{2} \sin ^{2}(\theta)+(R-r \cos (\theta))^{2}\right)^{\frac{3}{2}}}
$$




$$
\begin{aligned}
& =\frac{2 \pi}{R \bar{\alpha}} \int_{0}^{\pi} d \theta \frac{\sin (\theta)}{\left(\sin ^{2}(\theta)+\left(\frac{1}{\bar{\alpha}}-\cos (\theta)\right)^{2}\right)^{\frac{3}{2}}} \\
& =\frac{2 \pi}{R \bar{\alpha}} \frac{2 \bar{\alpha}^{3}}{\left(1-\bar{\alpha}^{2}\right)} .
\end{aligned}
$$

With this estimate we have proven that

$$
\left\|e^{(n)}\right\|_{L^{\infty}\left(\partial B_{R}\right)} \leqslant\left\|e^{(n-1)}\right\|_{L^{\infty}\left(\partial B_{r}\right)} \frac{\left(R^{2}-r^{2}\right)}{4 \pi r} \frac{2 \pi}{R \bar{\alpha}} \frac{2 \bar{\alpha}^{3}}{1-\bar{\alpha}^{2}}=\bar{\alpha}\left\|e^{(n-1)}\right\|_{L^{\infty}\left(\partial B_{r}\right)} .
$$

Now, we verify that $\int_{B_{R}} \mu_{k} \nabla e^{(n)} \cdot \nabla \varphi d x=0$ for every $\varphi \in H_{0}^{1}\left(B_{R}\right)$ and consequently that we have $\operatorname{div}\left(\mu_{k} \boldsymbol{\nabla} e^{(n)}\right)=0$ in $B_{R}$. Moreover

$$
\left|e^{(n)}(\boldsymbol{x})\right| \leqslant\left\|e^{(n)}\right\|_{L^{\infty}\left(\partial B_{R}\right)} \leqslant \bar{\alpha}\left\|e^{(n-1)}\right\|_{L^{\infty}\left(\partial B_{r}\right)} \quad \text { for } \boldsymbol{x} \in \partial B_{R} .
$$

By using a maximum principle (see [11] for instance), we obtain

$$
\left\|e^{(n)}\right\|_{L^{\infty}\left(B_{R}\right)} \leqslant \bar{\alpha}\left\|e^{(n-1)}\right\|_{L^{\infty}\left(\partial B_{R}\right)} \leqslant \bar{\alpha}\left\|e^{(n-1)}\right\|_{L^{\infty}\left(B_{R}\right)}=\frac{r}{R}\left\|e^{(n-1)}\right\|_{L^{\infty}\left(B_{R}\right)} .
$$

The proof is complete.

Remark 9. By using the relation (19), we prove that $\left\|e^{(n)}\right\|_{H^{1 / 2}\left(\partial B_{R}\right)}$ is bounded by $\left\|e^{(n-1)}\right\|_{L^{\infty}\left(\partial B_{r}\right)}$ and there exists a constant $C$ such that

$$
\left\|e^{(n)}\right\|_{H^{1}\left(B_{R}\right)} \leqslant C\left\|e^{(n-1)}\right\|_{L^{\infty}\left(\partial B_{r}\right)} .
$$

It follows that

$$
\left\|e^{(n)}\right\|_{H^{1}\left(B_{R}\right)} \leqslant C\left\|e^{(n-1)}\right\|_{L^{\infty}\left(B_{R}\right)} .
$$

Theorem 3 together with the definition of $e^{(n)}$ implies that there exists a constant again denoted by $C$ such that

$$
\left\|\widetilde{\psi}_{k+1}-\varkappa^{(n)}\right\|_{H^{1}\left(B_{R}\right)} \leqslant C\left(\frac{r}{R}\right)^{n}
$$

\section{Approximation in finite-dimensional space}

Let $W_{h} \subset W^{1}\left(\mathbb{R}^{3}\right) \cap W^{1, \infty}(\Omega)$ be a finite-dimensional subspace, of $W^{1}\left(\mathbb{R}^{3}\right) \cap W^{1, \infty}(\Omega)$, where

$$
W^{1, \infty}(\Omega)=\left\{g: \Omega \rightarrow \mathbb{R} \text { s.t. } \nabla g \in L^{\infty}(\Omega)\right\} .
$$

By using the same arguments we used for the proof of the existence and uniqueness of the solution $\psi \in W^{1}\left(\mathbb{R}^{3}\right)$ to the exact problem, we can prove that there exists a unique solution $\psi_{h} \in W_{h}$ satisfying

$$
\int_{\mathbb{R}^{3}} \bar{\mu}_{r}\left(\cdot,\left|\boldsymbol{H}_{0}+\boldsymbol{\nabla} \psi_{h}\right|\right)\left(\boldsymbol{H}_{0}+\boldsymbol{\nabla} \psi_{h}\right) \cdot \nabla \varphi_{h} d x=0, \quad \forall \varphi_{h} \in W_{h}
$$

Let us consider the mapping $F_{h}: W_{h} \rightarrow W_{h}$ defined by

$$
\int_{\mathbb{R}^{3}} \bar{\mu}_{r}\left(\cdot,\left|\boldsymbol{H}_{0}+\boldsymbol{\nabla} z_{h}\right|\right)\left(\boldsymbol{H}_{0}+\nabla F_{h}\left(z_{h}\right)\right) \cdot \nabla \varphi_{h} d x=0, \quad \forall \varphi_{h}, z_{h} \in W_{h} .
$$

Clearly, $F_{h}$ is well-defined since Hypothesis (H1) implies that the bilinear form

$$
a_{z}(\omega, \varphi)=\int_{\mathbb{R}^{3}} \bar{\mu}_{r}\left(\cdot,\left|\boldsymbol{H}_{0}+\boldsymbol{\nabla} z\right|\right) \boldsymbol{\nabla} \omega \cdot \nabla \varphi d x
$$


is continuous and coercive on $W_{h}$ for every $z \in W_{h}$ and

$$
L_{z}(\varphi)=\int_{\mathbb{R}^{3}} \bar{\mu}_{r}\left(\cdot,\left|\boldsymbol{H}_{0}+\nabla z\right|\right) \boldsymbol{H}_{0} \cdot \nabla \varphi d x
$$

is a continuous linear form for every $z \in W_{h}$.

Moreover, we have

$$
\left\|\nabla F_{h}(z)\right\|_{L^{2}\left(\mathbb{R}^{3}\right)} \leqslant \beta\left\|\mathbf{H}_{0}\right\|_{L^{2}\left(\mathbb{R}^{3}\right)}, \quad \forall z \in W_{h},
$$

and $\psi_{h}$ is the solution to Problem (21) if and only if $\psi_{h}$ is a fixed point of $F_{h}$ in $W_{h}$, i.e.,

$$
\psi_{h} \in W_{h} \text { satisfies } \psi_{h}=F_{h}\left(\psi_{h}\right)
$$

In order to compute the solution $\psi_{h}$ to Problem (21), we will use the following fixed point method

$$
\psi_{h}^{k+1}=F_{h}\left(\psi_{h}^{k}\right), \quad k=0,1,2, \ldots
$$

which is equivalent to solving at each step the finite dimensional linear problems: find $\psi_{h}^{k+1} \in W_{h}$ s.t.

$$
a_{\psi_{h}^{k}}\left(\psi_{h}^{k+1}, \varphi_{h}\right)=L_{\psi_{h}^{k}}\left(\varphi_{h}\right), \quad \forall \varphi_{h} \in W_{h}, \quad k=0,1,2, \ldots
$$

when $\psi_{h}^{0} \in W_{h}$ is given.

Remark 10. Algorithm (22) is the discrete version of the relaxed fixed point method (11)-(12) of Section 4 in which we set $\epsilon=1$. It follows that for solving the discrete problems $(22)$ in $W_{h}$, we can use a domain decomposition method that is analogous to the one given in Section 5. To do this, we discretize only $B_{R}$ (and not $\mathbb{R}^{3}$ ) with a tetrahedral mesh for instance, and use a standard finite element method for solving the discrete version of (17).

In the following, we introduce the inner product in $W_{h}$,

$$
((u ; w))_{\psi_{h}}=\int_{\mathbb{R}^{3}} \bar{\mu}_{r}\left(\cdot,\left|\boldsymbol{H}_{0}+\nabla \psi_{h}\right|\right) \boldsymbol{\nabla} u \cdot \nabla w d x
$$

and the norm $\|u\|_{\psi_{h}}=((u ; u))_{\psi_{h}}^{1 / 2}$ which is equivalent to every norm in the finite dimensional space $W_{h}$.

We reinforce Hypothesis (H1) by setting

$(\widetilde{\mathrm{H}} 1) \mu_{r}$ is a $C^{2}$ decreasing function on $[0, \infty)$ satisfying for every $s \in[0, \infty)$ :

1) $\beta^{\prime} \leqslant \mu_{r}(s) \leqslant \beta$,

2) $\mu_{r}(s)+s \mu_{r}^{\prime}(s) \geqslant \alpha>0$,

3) $\left|\mu_{r}^{\prime \prime}(s)\right| \leqslant \gamma$, and $\mu_{r}^{\prime}(0)=0$,

where $\gamma$ is a non-negative constant.

Observe that the first two hypotheses imply

$$
\lim _{s \rightarrow \infty}\left|\mu_{r}^{\prime}(s)\right|=\lim _{s \rightarrow \infty}\left(-\mu_{r}^{\prime}(s)\right) \leqslant \lim _{s \rightarrow \infty} \frac{\mu_{r}(s)-1}{s}=0,
$$

and the third hypothesis leads to

$$
\lim _{s \rightarrow 0} \frac{\left|\mu_{r}^{\prime}(s)\right|}{s}=\lim _{s \rightarrow 0} \frac{\left|\mu_{r}^{\prime}(s)-\mu_{r}^{\prime}(0)\right|}{s}=\mu_{r}^{\prime \prime}(0) .
$$

Thus there exists a positive constant $\lambda$ such that

$$
\frac{\left|\mu_{r}^{\prime}(s)\right|}{s} \leqslant \lambda, \quad \forall s \in[0, \infty) .
$$

Theorem 4. Assume that $\boldsymbol{H}_{0} \in L^{2}\left(\mathbb{R}^{3}\right) \cap L^{\infty}\left(\mathbb{R}^{3}\right)$ and that Hypothesis $(\widetilde{\mathrm{H}} 1)$ is satisfied, and let $\psi_{h} \in W_{h}$ be the unique solution to Problem (21). Then there exists $\kappa>0$ such that if $\left\|\psi_{h}-\psi_{h}^{0}\right\|_{\psi_{h}} \leqslant \kappa$, then $k \rightarrow \infty\left\|\psi_{h}-\psi_{h}^{k}\right\|_{\psi_{h}}=0$. 
Before proving this theorem, we give two results that can be easily verified.

Lemma 3. Let $f: \mathbb{R}^{3} \rightarrow \mathbb{R}$ be the mapping defined by $f(\boldsymbol{\xi})=\mu_{r}(|\boldsymbol{\xi}|)$. If $\boldsymbol{\xi}, \boldsymbol{\eta}, \boldsymbol{\zeta} \in \mathbb{R}^{3}, \boldsymbol{\xi} \neq \mathbf{0}$, then we have

$$
\begin{aligned}
& f^{\prime}(\boldsymbol{\xi}) \boldsymbol{\eta}=\mu_{r}^{\prime}(|\boldsymbol{\xi}|) \frac{\boldsymbol{\xi} \cdot \boldsymbol{\eta}}{|\boldsymbol{\xi}|} \\
& f^{\prime \prime}(\boldsymbol{\xi})(\boldsymbol{\eta}, \boldsymbol{\zeta})=\mu_{r}^{\prime \prime}(|\boldsymbol{\xi}|) \frac{\boldsymbol{\xi} \cdot \boldsymbol{\eta}}{|\boldsymbol{\xi}|} \frac{\boldsymbol{\xi} \cdot \boldsymbol{\zeta}}{|\boldsymbol{\xi}|}+\mu_{r}^{\prime}(|\boldsymbol{\xi}|)\left(\frac{\boldsymbol{\zeta} \cdot \boldsymbol{\eta}}{|\boldsymbol{\xi}|}-\frac{\boldsymbol{\xi} \cdot \boldsymbol{\eta} \boldsymbol{\xi} \cdot \boldsymbol{\zeta}}{|\boldsymbol{\xi}|^{2}} \frac{|\boldsymbol{\xi}|}{\mid{ }^{\prime}}\right)
\end{aligned}
$$

Lemma 4. Assume the hypotheses of Lemma 3 hold. Then

$$
\left|f^{\prime \prime}(\boldsymbol{\xi})(\boldsymbol{\eta}, \boldsymbol{\zeta})\right| \leqslant(\gamma+2 \lambda)|\boldsymbol{\zeta}||\boldsymbol{\eta}|
$$

Proof of Theorem 4. Let $\varphi_{h} \in W_{h}$ be given and compute $\phi_{h}=F_{h}\left(\psi_{h}+\varphi_{h}\right)-F_{h}\left(\psi_{h}\right)=F_{h}\left(\psi_{h}+\varphi_{h}\right)-\psi_{h}$. Thus $\phi_{h}+\psi_{h}=F_{h}\left(\psi_{h}+\varphi_{h}\right)$ and

$$
\int_{\mathbb{R}^{3}} \bar{\mu}_{r}\left(\cdot,\left|\boldsymbol{H}_{0}+\nabla\left(\psi_{h}+\varphi_{h}\right)\right|\right)\left(\boldsymbol{H}_{0}+\nabla\left(\psi_{h}+\phi_{h}\right)\right) \cdot \nabla z_{h} d x=0, \quad \forall z_{h} \in W_{h}
$$

By taking $z_{h}=\phi_{h}$, it follows that

$$
\begin{aligned}
\left\|\phi_{h}\right\|_{\psi_{h}}^{2} & =\left(\left(\phi_{h} ; \phi_{h}\right)\right)_{\psi_{h}}=\int_{\mathbb{R}^{3}} \bar{\mu}_{r}\left(\cdot,\left|\boldsymbol{H}_{0}+\boldsymbol{\nabla} \psi_{h}\right|\right)\left|\boldsymbol{\nabla} \phi_{h}\right|^{2} d x \\
& =\int_{\mathbb{R}^{3}}\left\{\bar{\mu}_{r}\left(\cdot,\left|\boldsymbol{H}_{0}+\boldsymbol{\nabla} \psi_{h}\right|\right)-\bar{\mu}_{r}\left(\cdot,\left|\boldsymbol{H}_{0}+\boldsymbol{\nabla}\left(\psi_{h}+\varphi_{h}\right)\right|\right)\right\}\left\{\left|\boldsymbol{\nabla} \phi_{h}\right|^{2}+\left(\boldsymbol{H}_{0}+\boldsymbol{\nabla} \psi_{h}\right) \cdot \boldsymbol{\nabla} \phi_{h}\right\} d x \\
& =\int_{\Omega}\left\{\mu_{r}\left(\left|\boldsymbol{H}_{0}+\boldsymbol{\nabla} \psi_{h}\right|\right)-\mu_{r}\left(\left|\boldsymbol{H}_{0}+\boldsymbol{\nabla}\left(\psi_{h}+\varphi_{h}\right)\right|\right)\right\}\left\{\left|\boldsymbol{\nabla} \phi_{h}\right|^{2}+\left(\boldsymbol{H}_{0}+\boldsymbol{\nabla} \psi_{h}\right) \cdot \boldsymbol{\nabla} \phi_{h}\right\} d x .
\end{aligned}
$$

By using a Taylor expansion and Lemma 4, we obtain

$$
\begin{aligned}
\left\|\phi_{h}\right\|_{\psi_{h}}^{2} \leqslant & \int_{\Omega}\left\{\left|\mu_{r}^{\prime}\left(\left|\boldsymbol{H}_{0}+\boldsymbol{\nabla} \psi_{h}\right|\right)\right|\left|\boldsymbol{\nabla} \varphi_{h}\right|+\frac{1}{2}(\gamma+2 \lambda)\left|\nabla \varphi_{h}\right|^{2}\right\} \\
& \times\left\{\left|\boldsymbol{\nabla} \phi_{h}\right|^{2}+\left|\boldsymbol{H}_{0}+\boldsymbol{\nabla} \psi_{h}\right| \cdot\left|\nabla \phi_{h}\right|\right\} d x .
\end{aligned}
$$

Using Hypothesis $(\widetilde{\mathrm{H}} 1)$, i.e., $0 \leqslant-s \mu_{r}^{\prime}(s) \leqslant \mu_{r}(s)-\alpha$, we obtain by setting $\sigma=\sup _{s \in[0, \infty)}\left|\mu_{r}^{\prime}(s)\right|$ :

$$
\begin{aligned}
\left\|\phi_{h}\right\|_{\psi_{h}}^{2} \leqslant & \int_{\Omega} \mu_{r}\left(\left|\boldsymbol{H}_{0}+\boldsymbol{\nabla} \psi_{h}\right|-\alpha\right)\left|\boldsymbol{\nabla} \varphi_{h}\right|\left|\boldsymbol{\nabla} \phi_{h}\right| d x+\sigma \int_{\Omega}\left|\boldsymbol{\nabla} \varphi_{h}\right|\left|\boldsymbol{\nabla} \phi_{h}\right|^{2} d x \\
& +\int_{\Omega} \frac{1}{2}(\gamma+2 \lambda)\left|\boldsymbol{\nabla} \varphi_{h}\right|^{2}\left|\boldsymbol{\nabla} \phi_{h}\right|^{2} d x \\
& +\int_{\Omega} \frac{1}{2}(\gamma+2 \lambda)\left|\nabla \varphi_{h}\right|^{2}\left|\boldsymbol{H}_{0}+\nabla \psi_{h}\right| \cdot\left|\nabla \phi_{h}\right| d x
\end{aligned}
$$

Therefore, there exists a positive constant $C$ such that

$$
\begin{aligned}
\left\|\phi_{h}\right\|_{\psi_{h}}^{2} \leqslant & \int_{\Omega} \mu_{r}\left(\left|\boldsymbol{H}_{0}+\boldsymbol{\nabla} \psi_{h}\right|\right)\left(1-\frac{\alpha}{\mu_{r}\left(\left|\boldsymbol{H}_{0}+\boldsymbol{\nabla} \psi_{h}\right|\right)}\right)\left|\boldsymbol{\nabla} \varphi_{h}\right|\left|\boldsymbol{\nabla} \phi_{h}\right| d x \\
& +C\left\|\boldsymbol{\nabla} \varphi_{h}\right\|_{L^{\infty}(\Omega)}\left\|\nabla \phi_{h}\right\|_{L^{\infty}(\Omega)}\left(\left\|\boldsymbol{\nabla} \varphi_{h}\right\|_{L^{\infty}(\Omega)}+\left\|\nabla \phi_{h}\right\|_{L^{\infty}(\Omega)}+\left\|\nabla \varphi_{h}\right\|_{L^{\infty}(\Omega)}\left\|\nabla \phi_{h}\right\|_{L^{\infty}(\Omega)}\right) .
\end{aligned}
$$

By using the inequality $\mu_{r}(s) \leqslant \beta$ and the equivalence of the norms in $W_{h}$, there exists another constant $D$ such that

$$
\begin{aligned}
\left\|\phi_{h}\right\|_{\psi_{h}}^{2} \leqslant & \left(1-\frac{\alpha}{\beta}\right) \int_{\mathbb{R}^{3}} \bar{\mu}_{r}\left(\cdot,\left|\boldsymbol{H}_{0}+\nabla \psi_{h}\right|\right)\left|\nabla \varphi_{h}\right|\left|\boldsymbol{\nabla} \phi_{h}\right| d x \\
& +D\left\|\varphi_{h}\right\|_{\psi_{h}}\left\|\phi_{h}\right\|_{\psi_{h}}\left(\left\|\varphi_{h}\right\|_{\psi_{h}}+\left\|\phi_{h}\right\|_{\psi_{h}}+\left\|\varphi_{h}\right\|_{\psi_{h}}\left\|\phi_{h}\right\|_{\psi_{h}}\right)
\end{aligned}
$$




$$
\begin{aligned}
\leqslant & \left(1-\frac{\alpha}{\beta}\right)\left\|\varphi_{h}\right\|_{\psi_{h}}\left\|\phi_{h}\right\|_{\psi_{h}} \\
& +D\left\|\varphi_{h}\right\|_{\psi_{h}}\left\|\phi_{h}\right\|_{\psi_{h}}\left(\left\|\varphi_{h}\right\|_{\psi_{h}}+\left\|\phi_{h}\right\|_{\psi_{h}}+\left\|\varphi_{h}\right\|_{\psi_{h}}\left\|\phi_{h}\right\|_{\psi_{h}}\right) .
\end{aligned}
$$

It follows that

$$
\left\|\phi_{h}\right\|_{\psi_{h}} \leqslant\left(1-\frac{\alpha}{\beta}\right)\left\|\varphi_{h}\right\|_{\psi_{h}}+D\left\|\varphi_{h}\right\|_{\psi_{h}}\left(\left\|\varphi_{h}\right\|_{\psi_{h}}+\left\|\phi_{h}\right\|_{\psi_{h}}+\left\|\varphi_{h}\right\|_{\psi_{h}}\left\|\phi_{h}\right\|_{\psi_{h}}\right) .
$$

From this last relation, it is easy to prove that there exists $\kappa>0$ such that if $\left\|\varphi_{h}\right\|_{\psi_{h}}<\kappa$ then

$$
\left\|\phi_{h}\right\|_{\psi_{h}} \leqslant\left(1-\frac{\alpha}{2 \beta}\right)\left\|\varphi_{h}\right\|_{\psi_{h}} .
$$

If we set $\varphi_{h}=\psi_{h}^{k}-\psi_{h}$, we have $\phi_{h}=\psi_{h}^{k+1}-\psi_{h}$, and using the above relation we obtain

$$
\left\|\psi_{h}^{k}-\psi_{h}\right\|_{\psi_{h}} \leqslant \kappa\left(1-\frac{\alpha}{2 \beta}\right)^{k}
$$

When $\psi_{h}^{0} \in W_{h}$ is chosen such that $t\left\|\psi_{h}^{0}-\psi_{h}\right\|_{\psi_{h}}<\kappa$, the conclusion follows.

\section{$7 \quad$ Numerical results}

In Section 4, we have seen that the relaxed fixed point method is converging when $\epsilon<2 / \beta$, independently of the starting point $\psi_{0}$ in $W^{1}\left(\mathbb{R}^{3}\right.$ ) (see Theorem 2). In our physical applications, $\beta=4000$ (see Figure 2) and $\epsilon=\frac{2}{\beta}$ is very small. Following this result, the convergence of $\psi_{k}$ to $\psi$, when $k$ tends to infinity, is very slow since $\psi_{k+1}$ is almost egal to $\psi_{k}$.

However, in the finite-dimensional situation of Section 6 , the convergence of $\psi_{h}^{k}$ to $\psi_{h} \approx \psi$ when $k$ tends to infinity is faster since we can take $\epsilon=1$ (see Theorem 4). However, in this case, we have to take $\psi_{h}^{0}$ very close to $\psi_{h}$. Actually, our result of convergence holds when $\left\|\psi_{h}-\psi_{h}^{0}\right\|_{\psi_{h}} \leqslant \kappa$, where $\kappa$ could depend on the finite space $W_{h} \subset W^{1}\left(\mathbb{R}^{3}\right)$. The theoretical results of Sections 4 and 5 tends to claim that $\kappa$ converges to zero when $W_{h}$ becomes dense to the limit in $W^{1}\left(\mathbb{R}^{3}\right)$. The following numerical results show that, in fact, this is not the case and that the algorithm presented in Section 4 on the continuous problem is converging even if $\epsilon=1$. In other words, it seems that, for every starting point $\psi_{0}$, the fixed point algorithm

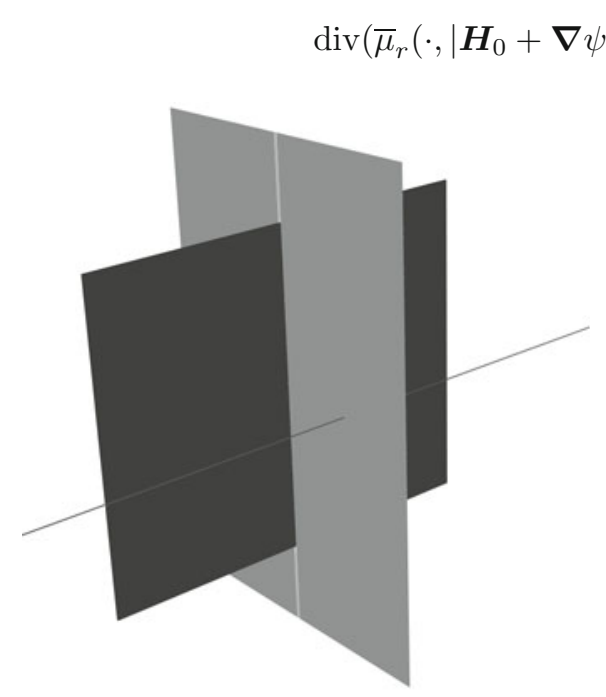

Figure 1 Geometry of the test-case with the plate in black, the observation plane in gray and the support of electrical current in line

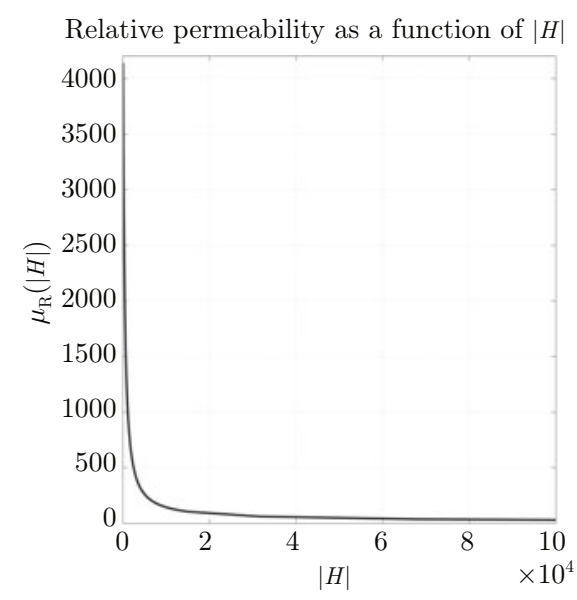

Figure 2 Relative permeability $\mu_{r}(|\boldsymbol{H}|)$ as a function of $|\boldsymbol{H}|$ 

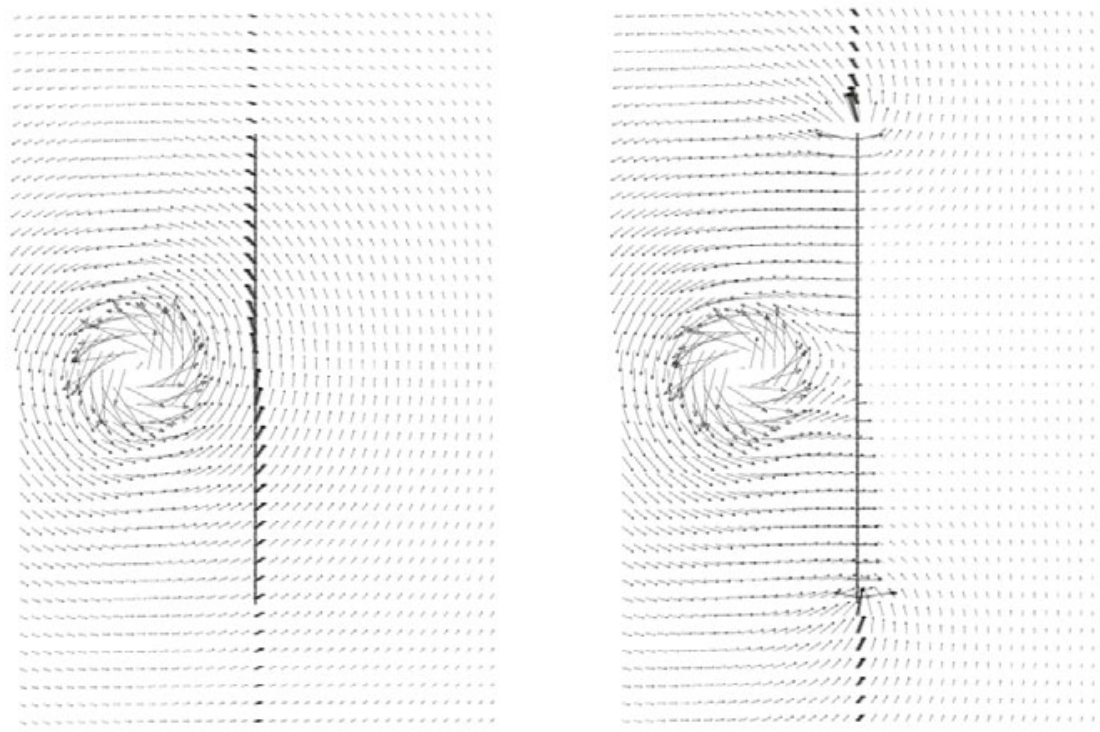

Figure 3 Magnetic field in the observation plane as if the plate where not there (left) and with the plate (right). We can observe the screening effect behind the plate
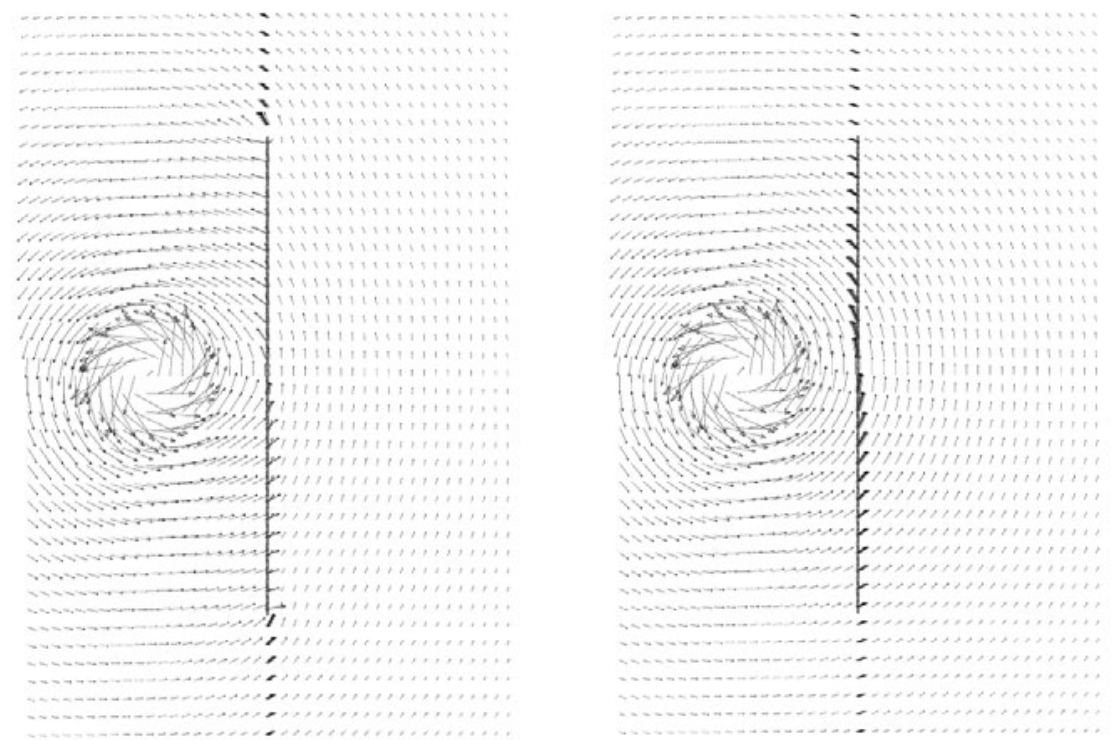

Figure 4 Magnetic field $\boldsymbol{H}$ in the observation plane for a current of $10^{6} \mathrm{~A}$ (left) and for a current of $10^{8} \mathrm{~A}$ (right). We can observe the saturation of the plate for high current

is converging.

This problem is open. In order to support this, we will consider a ferromagnetic rectangular plate $\Omega, 5$ meters wide, 4 meters high and 2 centimeters thick, which is placed in front of an idealized infinitely long wire with zero section. The origin $O$ is placed at the center of the plate; the axis $O x_{1}$ is in the direction of the thickness of the plate; the axis $O x_{2}$ is in the direction of the length and $O x_{3}$ is in the direction of the height of the plate. The total electric current of $1 \mathrm{~A}$ flows in the wire parallel to $O x_{2}$ and passes through the point $(1.01,0,0)$ (see Figure 1). The relative magnetic permeability $\mu_{r}$ as a function of $|\boldsymbol{H}|$ is given in Figure 2. In Figure 1 we also represent the observation plane we have chosen to observe the screening effect behind the plate when we go away perpendicularly from it. The small ball $B_{r}$ is with radius $r=3.5$ meters centered at the origin $O$ and the radius of the ball $B_{R}$ is $R=4.4$ meters.

In order to build the finite-dimensional space $W_{h}$, we use a finite element method with piecewise 
polynomials of degree 1 on a tetrahedral mesh $\Upsilon_{h}$ discretizing the big ball $B_{R}$. We solve the approximate problem set in the whole space $\mathbb{R}^{3}$ by using the finite-dimensional version of the domain decomposition algorithm presented in Section 6.

Figure 3(left), shows on the observation plane the magnetic field $\boldsymbol{H}_{0}$ as if the plate were not present. Because the current support is perpendicular to the observation plane, the magnetic field is parallel to that plane. Figure 3(right) shows on the observation plane the magnetic field $\boldsymbol{H}$ at the same scale. We can observe the screening effect behind the plate. Moreover, we can observe that near the plate boundary, the magnetic field is perpendicular to the plate when outside the plate and parallel to the plane of the plate when inside the plate. This is due to the jump of $\mu_{r}$ across the plate boundary with continuity of $\boldsymbol{B} \cdot \boldsymbol{n}$ and of $\boldsymbol{H} \times \boldsymbol{n}$.

Figure 4 shows $\boldsymbol{H}$ in the observation plane for different values of the current: the current is $10^{6} \mathrm{~A}$ on the left figure and $10^{8} \mathrm{~A}$ on the right figure. We can see the saturation effect: with a current of $10^{8} \mathrm{~A}$, the plate has no screening effect anymore. This can also be observed with $\mu_{r}$ : for a current of $1 \mathrm{~A}$, the relative permeability in the plate is almost constant to 4136 ; for a current of $10^{8} \mathrm{~A}, \mu_{r}$ is almost constant to 106.

Acknowledgements This work was supported by Rio Tinto-Alcan Company. The authors would like to thank Bernard Dacorogna for fruitful discussions about calculus of variations.

\section{References}

1 Barrett J W, Liu W. Finite element approximation of degenerate quasilinear elliptic and parabolic problems. In: Pitman Research Notes in Mathematics Series, 303. Harlow: Longman, 1994, 1-16

2 Barrett J W, Liu W. Quasi-norm error bounds for the finite element approximation of a non-Newtonian flow. Numer Math, 1994, 68: 437-456

3 Chadebec O, Colomb J L, Janet F. A review of magnetostatic moment method. IEEE Trans Magn, 2006, 42: 515-520

4 Dacorogna B. Direct Methods in the Calculus of Variations. Applied Mathematical Sciences, 78. Berlin: SpringerVerlag, 1989

5 Dautray R, Lions J L. Mathematical Analysis and Numerical Methods for Science and Technology. Berlin: SpringerVerlag, 1990

6 Descloux J, Flueck M, Rappaz J. Modelling and mathematical results arising from ferromagnetic problems. Mathicse, École Polytechnique Fédérale de Lausanne, Switzerland, 2010

7 Descloux J, Flueck M, Romerio M V. A problem of magnetostatics related to thin plates. Math Model Numer Anal, 1998, 32: 859-876

8 Friedman M. Mathematical study of the nonlinear singular integral magnetic field equations, I. SIAM J Appl Math, 1980, 39: 14-20

9 Friedman M. Mathematical study of the nonlinear singular integral magnetic field equations, II. SIAM J Number Anal, 1981, 18: 644-653

10 Friedman M. Mathematical study of the nonlinear singular integral magnetic field equations, III. SIAM J Math Anal, 1981, 12: 536-540

11 Gilbarg D, Trudinger N. Elliptic Partial Differential Equations of Second Order. Classics in Mathematics. Berlin: Springer-Verlag, 1988

12 Haddar H, Joly P. Effective boundary conditions for thin ferromagnetic layers: the one dimensional model. SIAM J Appl Math, 2001, 61: 1386-1417

13 Joly P, Vacus O. Mathematical and numerical studies of nonlinear ferromagnetic material. Math Model Numer Anal, 1999, 33: 593-626

14 Monk P B, Vacus O. Error estimates for a numerical scheme for ferromagnetic problems. SIAM J Numer Anal, 1999, 36: $696-718$ 